\title{
Graphic Models of Micelles of Solutions of Sodium Salts of Fatty Acids and Research of Changes occurring in them
}

\author{
S. S. Saidvaliev and K.Kh. Majidov* \\ Bukhara Engineering-Technological Institute, 15, K. Murtazaevstreet, \\ Bukhara city, Uzbekistan \\ *Corresponding author
}

A B S T R A C T

\begin{tabular}{l} 
K e y w o r d s \\
Fatty acids, Sodium \\
soaps, Micelles, \\
Graphical models, \\
Structural elements, \\
Diffusion layer, \\
Formation conditions, \\
Structure formation, \\
Fluidity, Aggregate \\
state, Thickness, Side \\
sections, Linear \\
dimensions \\
\hline $\begin{array}{l}\text { Article Info } \\
\text { Accepted: } \\
\text { 22 June } 2020 \\
\text { Available Online: } \\
\text { 10 July } 2020\end{array}$ \\
\hline
\end{tabular}

\section{Introduction}

At present, the features of the composition and structure of the salable appearance of bar soaps have not been studied sufficiently. Information has fragmented, contradictory nature and not related to practical tasks of soap production (Abramzonet.al, 1991; Rosen et al., 1996).

Present scientific conceptions are based on research of the properties of colloidal systems consisting of low-concentrated solutions of individual salts of fatty acids (Rosen et.al, 1996; Arutyunyan, 1996), and are seldom applicable to highly concentrated real systems consisting of mixtures of salts differing in the length of the hydrocarbon chain and saturation degree.

Research, systematization, analysis of existing concepts and development of formation structure of bar soaps, explaining structure and properties of industrial types of 
bar commercial soaps obtained based on sodium salts of fatty acids, are relevant

Recently great attention has been paid to improving technology and expanding the assortment bar solid household and toilet soaps (Saidvaliev et al., 2013; Saidvaliev et al., 2010). This requires the research and establishment of detergents, bar soaps used in practice (Saidvaliev et al., 2010; Saidvaliev et al., 2015).

Purpose of the work is aimed to creation graphic models of micelle of solutions of sodium salts of fatty acids and study changes occurring in them.

\section{Materials and Methods}

Hydrolysis methods were used for the preparation of graphic models of micelles of sodium soap solutions (Arutyunyan, 1998). The sizes of soap molecules are determined by macroscopic measurement of a solid body (Saidvaliev et al., 2010). Modern methods of analysis and estimation of structure formation of soap system have been used in research (Saidvaliev et al., 2015).

\section{Results and Discussion}

It has been experimentally established that characteristic feature of the industrial technology of bar soaps is the use of fat raw materials consisting of $60 \%$ saturated and $40 \%$ unsaturated fatty acids, as well as its processing according to three stages: alkaline hydrolysis of fat raw materials, cooling and dehydration of the obtained soap base, and mechanical treatment of soap shavings. Because of technological operations, which vary the combination concentrationtemperature-time, the state of the system changes from viscous-flow to crystal-like states.
At the first stage, during alkaline hydrolysis of prescription mixtures of fats and oils, disperse systems are formed, which consist of water and five basic sodium salts of fatty acids in soap fractions: lauric $^{0}{ }_{12}$, myristic $C_{14}^{0}$, palmitic $C_{16}^{0}$, stearic $\mathrm{C}_{18}^{0}$ and oleic $\mathrm{C}^{1=}{ }_{18}$ fractions in an approximate ratio: 1 0: 2.0: 6.0: 5.0: 9.0.

Compared to the size of soap molecules, which are several tens of angstroms, the saponated system in the volume of the soap boiler is a microscopic body that allows the existence of several transition states.

In the temperature range of the process 85$95^{\circ} \mathrm{C}$, the salt concentration is about 66 mass $\%$, which is several orders of magnitude higher than the CMC (critical micelle concentration), the value of which for these mixtures was less than one percent.

Thereby, mixed lamellar micelle can be represented as the main structural unit of the system.

Salts that are constituents of micelle have a different degree of saturation and the length of the hydrocarbon radical, and, therefore, different physical-chemical properties, the real surface of mixed micelles can be heterogeneous, have acurvature and differ from the plane.

In order to construct a correct graphical model of the micelle, it was necessary to approximate the projection of the section of micelle surface in a straight line, which was methodically accomplished by calculating the length of the hydrocarbon chain of each molecule and the "equivalent" length of the hydrocarbon chain of these molecules.

The lengths of the hydrocarbon chain of each type of molecule, which are the main ones in the composition of commodity toilet soaps, 
are calculated using the Tenford empirical formula, and are presented in table 1

$\mathrm{L}_{\mathrm{n}}=1.5+1.26 \mathrm{n}_{\mathrm{i}}$

Where, $\mathrm{n}_{\mathrm{i}}=-$ number of hydrocarbon atoms in the chain;

"Equivalent" length of the soap molecule, which made $21.6 \AA$, was calculated by the formula:

$$
L_{\ni}=\frac{\sum\left(m_{n} \cdot L_{n}\right)}{\sum m_{n}}
$$

where, $\mathrm{L}_{\ni}$ - "equivalent" length of the soap molecule, $\mathrm{m}_{\mathrm{n}}$ - mass fraction of acid salt $(\%)$, $\mathrm{L}_{\mathrm{n}}$-length of molecule of fatty acid salt $(\AA)$.

Aggregation of molecules into micelles and their decay is an equilibrium process of various opposite stages of association, and is characterized by relaxation time, i.e. time of complete or partial thermodynamic equilibrium of the aggregation process. The average residence time of the molecules in the soap micelle in the thermodynamic equilibrium state is about 10 seconds.

Then, the theoretical model of a mixed lamellar micelle of toilet soap in equilibrium state can be represented in the following form (Fig. 1).

The fact that, according to the presented model, the anions are in dynamic equilibrium with the micelle and, therefore, unlike the existing models, the plate is surrounded not with water, but with a solution of sodium salts of fatty acids, that be a part of micelle, is important for the development of further theoretical concepts. The composition of the solution differs from the composition of the micelle, and is determined by the degree of solubility of the individual salts.
The main structural element of the micelle is a flexible plate, which is constructed similarly to the lamellar micelle proposed by Mac Bane. The plate is formed as double row of diphilic molecules of sodium salts. The row consists of molecules located almost parallel to each other and perpendicular to the outer surface of the plate, the polar parts (carboxyl group) of which are on the upper and lower surfaces of the micelle in contact with the solution, and non-polar hydrocarbon radicals form their hydrophobic inner part that does not contain water.

The main parameters of the plate are the thickness of plate $d_{n}$ and specific surface $S_{n}$ occupied by the polar group; the plate length is considered to be indefinite or, if necessary, its numerical values equal to infinity.

The equivalent thickness of the plate is approximately equal to the length of two soap molecules with fully extended hydrocarbon chains, and is about $42 \AA$.

The specific surface of the carboxyl group is independent of the temperature and fatty acid composition of the soap, and is on average $S_{n}$ $39 \AA$.

Because of the dissociation of soap molecules according to the equation:

$\mathrm{RCOONa} \leftrightarrow \mathrm{RCOO}^{-}+\mathrm{Na}-+$

on the surface of the micelle plate double electric layer (DEL) is formed, which prevents the micelles from sticking together (Fig. 2).

Voltage-forming layer consists of anions of the carboxyl group $\mathrm{RCOO}^{-}$, and is negatively charged. Counter-ions are positively charged $\mathrm{Na}^{+}$ions. Because of adsorption, part of the $\mathrm{Na}^{+}$ions is in a fixed state below the A- $\AA$ sliding surface, the other part is a diffusion layer, the thickness of which depends on the 
temperature and concentration of soap molecules in the solution between the plates; in practical conditions may exceed $20 \AA$.

Obviously, in real conditions, the system will consist of micellar associates, the structure and properties of which are the object of independent research.

It is obvious that under real conditions, the system will consist of micellar associates, the structure and properties of which are the object of independent research.

One of the intermediate products of the industrial technology of bar soap is a soap base, which at temperature of $85-95{ }^{\circ} \mathrm{C}$ and moisture content of about $33 \%$ is a liquid-like dispersed system, which under the gravity force can flow without destruction, and consists of aggregated mixed lamellate micelles of anions (molecules) of sodium salts of fatty acids $\mathrm{C}_{12}-\mathrm{C}_{18}$ fractions.

Theoretical model of subsequent aggregation of lamellate micelles into the lamellar structure(Abramzonet.al, 1991), restricting the spatial dimensions of micelles only by their thickness $\mathrm{D}$ equal to double length of the monomer $(\mathrm{D}=21)$ at the length $\mathrm{L}$ and the width of micelles $\mathrm{H}$ tending to infinity $(\mathrm{L} \rightarrow$ $\infty, \mathrm{H} \rightarrow \infty)$, is argumentative, since it is seldom applicable to the real system operating with finite quantities.

It should be assumed that in a real structure, at least one other size parameter of the micelle must have a finite value relative to the unit of scaling, in this case, the thickness of the micelle.

Then the further structuring of the system can take place in one of the possible directions the formation of closed aggregates in two dimensions during the interaction of the lateral micelles. Moreover, the length La of the formed associate will be much larger than its cross section $P_{a}$, i.e.: $L_{a}>>P_{a}$. Initial stage of structuring can begin before the losses in system fluidity, and would be characterized by increase in the direct contact of the lateral micelles with each other or (more likely), contact through the layers of the dispersed medium.

Double electric layers are the least developed at the ends of the lateral sections, and action of molecular forces, causing the cohesion of micelles, is revealed exactly in these places.

Moreover, the process can take place simultaneously in two directions. In the first case, a closed branched structure is formed (Fig. 3), the side of the projection onto the plane of which is equal to the estimated micelle width.

In the second case, subsequent hardening of the end bonds can lead to the formation of spheroidal closed aggregates containing in the inner cavity the vesicles - solution of individual salts. If a micelle can be defined as a closed monolayer, then a vesicle is a closed bilayer.

The theoretical model of the vesicles is shown in Fig. 4.

Simultaneous formation of vesicles by the following mechanism is also possible. Adhesion of lamellate micelles is carried out in direction of increase of linear dimensions, at which length of micelle far exceeds its thickness. In this case, because of the mixed composition of the plates, bending fluctuation becomes significant, and if a bulge forms in the middle of the micelle, then the tensile force of linear tension at the edges will intensify it, because it is directed at reducing the perimeter of the micelle. As a result, the edges of the micelles are bonded and formed a vesicle (Fig.5) having a larger diameter than in the first case. 
Table.1 Lengths of the hydrocarbon chain of the molecules that are the main ones in the composition of commercial toilet soaps

\begin{tabular}{|l|l|l|l|l|l|}
\hline Acid index & $\mathbf{C}_{12}^{\mathbf{0}}$ & $\mathbf{C}^{\mathbf{0}}{ }_{\mathbf{1 4}}$ & $\mathbf{C}_{\mathbf{1 6}}^{\mathbf{0}}$ & $\mathbf{C}_{\mathbf{1 8}}^{\mathbf{0}}$ & $\mathbf{C}^{\mathbf{1}=} \mathbf{1 8}$ \\
\hline $\begin{array}{l}\text { Length of } \\
\text { hydrocarbon } \\
\text { chain A }\end{array}$ & 15.4 & 18.0 & 20.5 & 23.0 & 23.0 \\
\hline
\end{tabular}

Fig.1 Model of mixed lamellar micelle of toilet soap:

1- lamellar micelle with thickness $d_{n} \sim 42 \AA$; 2- solution of anions in molecularly dispersed state; 3 - anion of the soap molecule

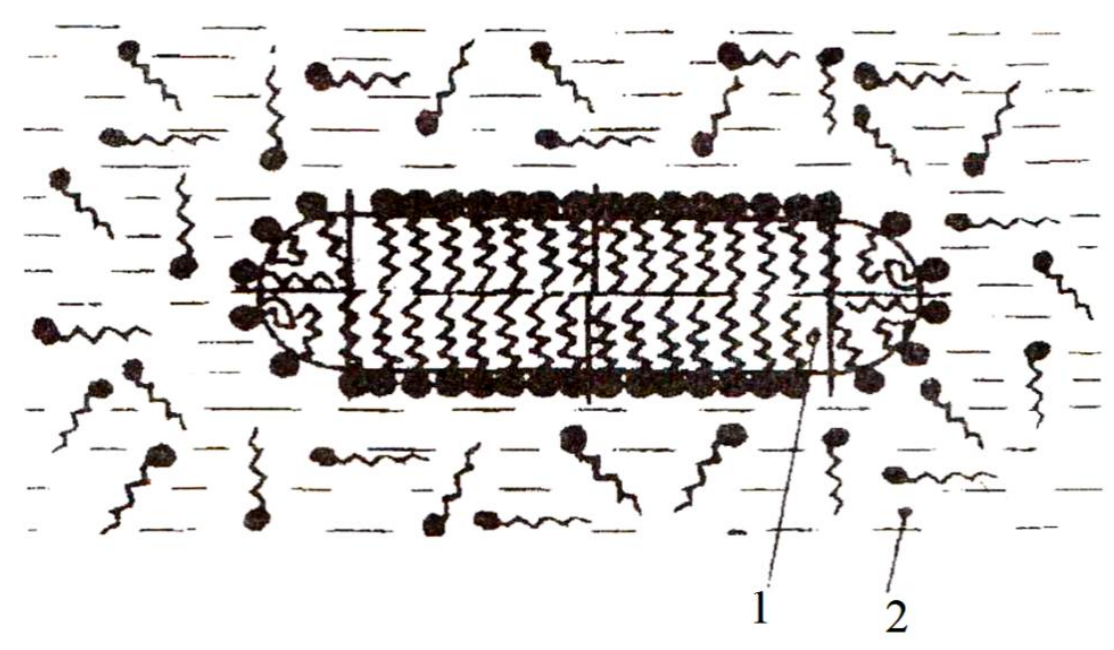

Fig.2 - anion of soap molecules; + ions of sodium;

A-A - sliding surface; $\mathrm{H}$ - thickness of diffusion layer, which is about $20 \AA$

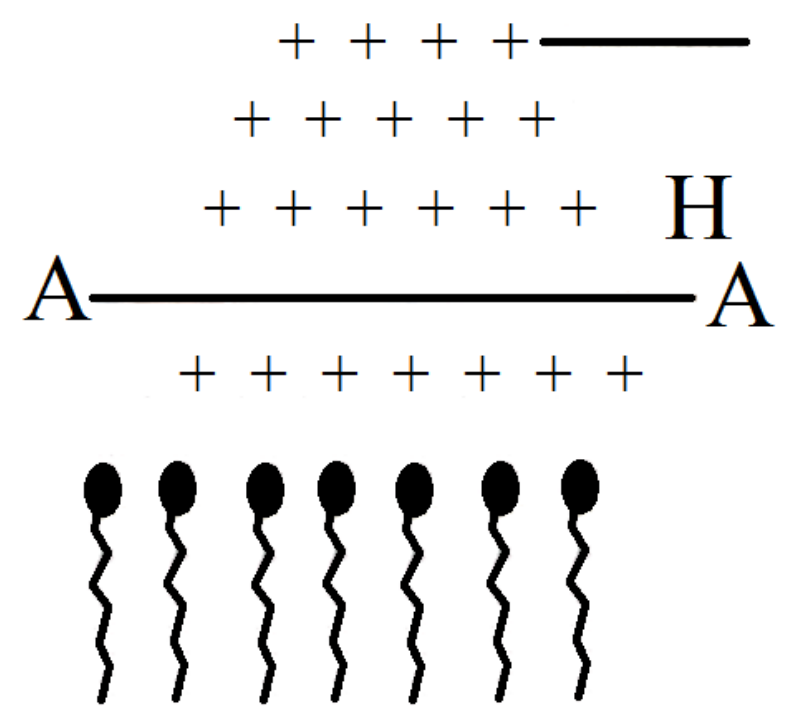


Fig.3 Structure of closed zones formed at the initial stage of structuring of soap base:

1- micelles of soap (dispersed phase); 2- surface areas of micelles with minimal development of the double electric layer; 3 - structure space filled with dispersion medium

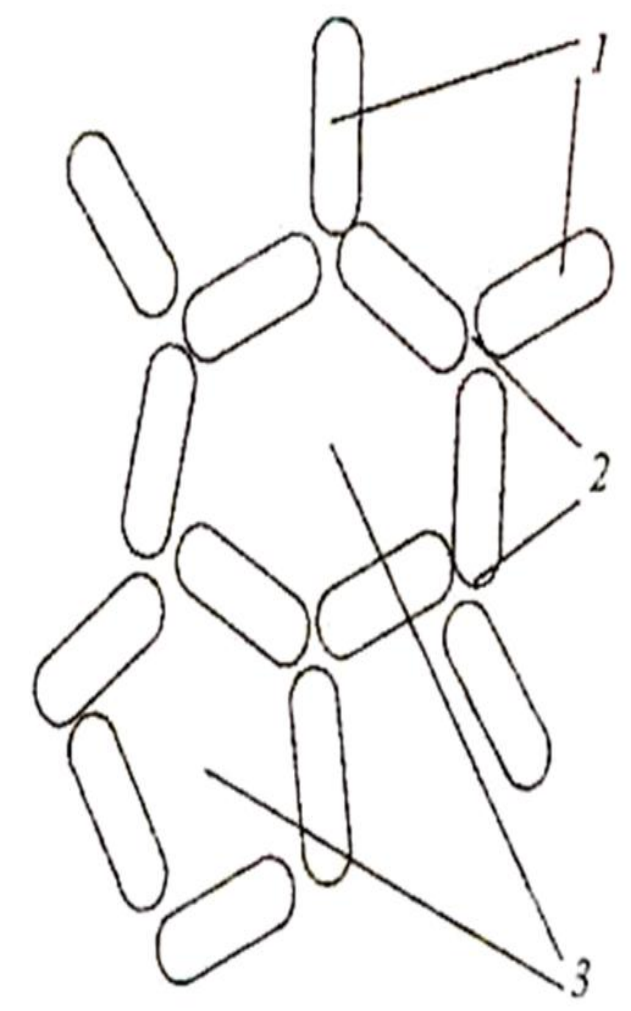

Fig.4 Aggregation of lamellate micelles in the closed system:

1- micelle, 2- solution of individual salts.

Where $r_{1}$ and $r_{2}$ - outer and inner radii of the vesicle, $h$-thickness of wall, $l_{1}$ and $l_{2}-$ the thickness of the outer and inner layers

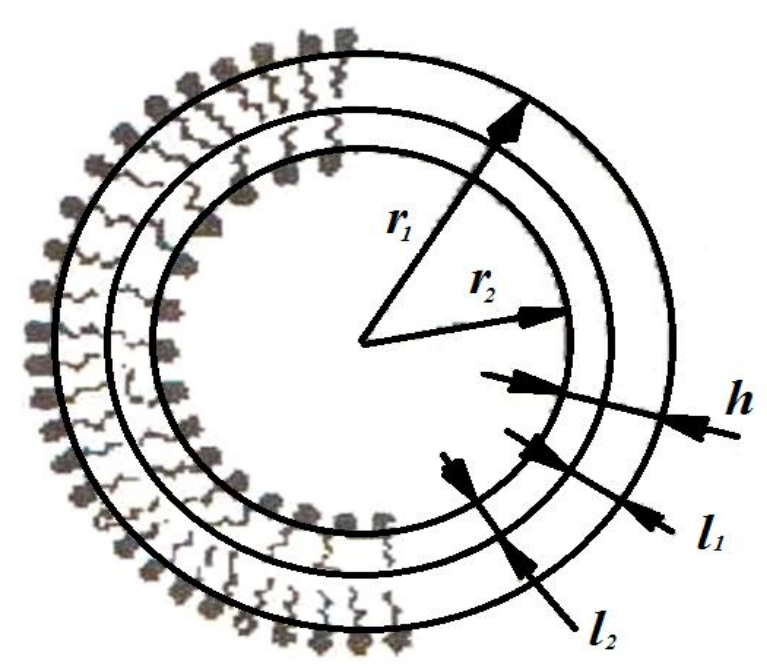


Fig.5 Formation of vesicles from elongated lamellate micelles

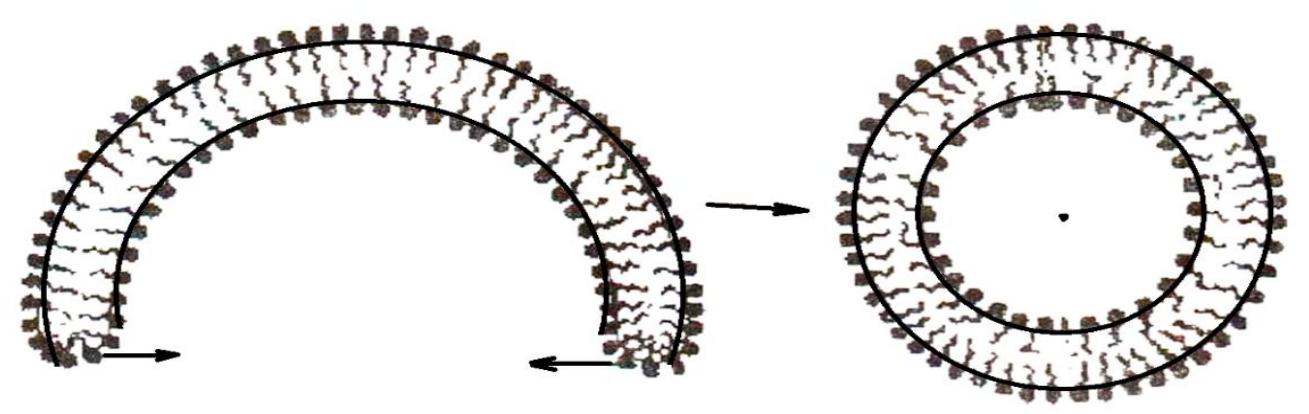

Fig.6 Hexagonal packed cylindrical vesicles

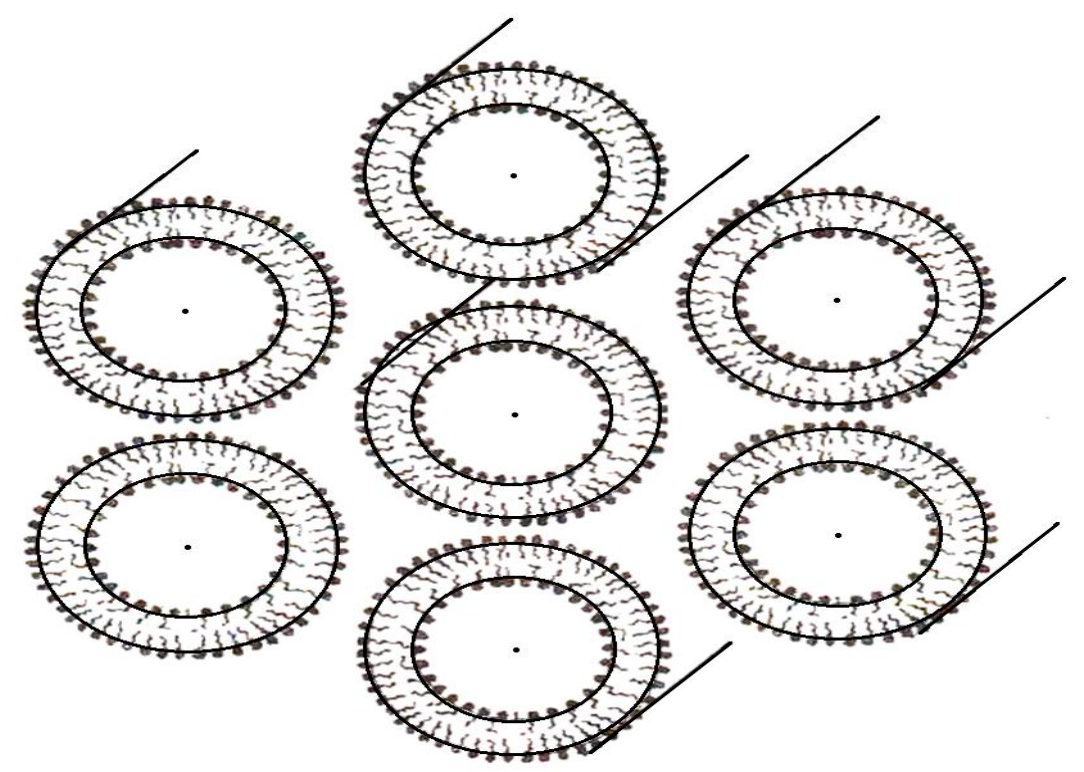

In both the first and second cases, the subsequent approach of the spherical vesicles can form a system of hexagonal packed cylindrical vesicles (Fig. 6).

Then, in general case, in the temperature range $95-70^{\circ} \mathrm{C}$ the soap base can be represented as a transition zone characterized by two or more transformation levels of mixed plate micelles - from the beginning of lateral interactions to the stage of formation of hexagonal packed cylindrical vesicles of various diameters, containing solution of salts of fatty acid both in the internal cavities of the vesicles and in the intervisicular space.
In conclusion, analysis and evaluation of available theoretical representations allowed establishing graphical models of micelles of solutions of fatty acids sodium salts in production of varieties of bar household and toilet soaps. Studying the features of structure formation of micelle of sodium salts of fatty acids of bar soap varieties allows determining and establishing their detergent properties in various technological stages of utilization.

\section{References}
Abramzon
A.L.
Grigoryev
S.M., Makaronova N.N. Influence of 
electrostatic interactions on the adsorption of ionic surface-active materials on liquid surfaces / Collected articles of All-Union seminar on colloid chemistry of food and bioactive disperse systems. M.: Nauka, 1991, p. 7-22.

Rosen M., Liu L. Surface Activity and Premicellar Aggregation of Some NoveeDigyatemary Gemini Surfactants // JAOCS. 1996. V.73. N 7. P.885-890.

Arutyunyan N.S. Technology of fat processing. M.: Pishepromizdat, 1998, p. 248

Saidvaliev, S. S., A. M. Rakhimov, K. Kh. Majidov "Expanding the assortment and improving the quality of varieties of toilet soap" Scientific-practical conference, Bukhara, 2013.

Saidvaliev, S.S., A.M. Rakhimov, M.N.

Rakhimov, K.Kh. Majidov

Developments to create new types of toilet soaps and improve their quality // Journal "Chemistry and chemical technology", - 2010, No. 30, p. 2.

Saidvaliev, S.S., M.N. Rakhimov, K.Kh. Majidov, Development of new technologies for production of toilet and household varieties of soap // Materials of $10^{\text {th }}$ International Conference "Oil and fat industry2010" St. Petersburg. 2010, p. 112

Saidvaliev, S. S., M. M. Mamatov, K. Kh. Majidov "Research of the surface tension of soap solutions" Oil and fat industry, 2015.

\section{How to cite this article:}

Saidvaliev, S. S. and Majidov, K.Kh. 2020. Graphic Models of Micelles of Solutions of Sodium Salts of Fatty Acids and Research of Changes occurring in them. Int.J.Curr.Microbiol.App.Sci. 9(07): 2923-2930. doi: https://doi.org/10.20546/ijcmas.2020.907.344 